\section{A More Accurate Explicit Scheme to Solve Certain Quantum Operator Equations of Motion}

\section{Luis Vázquez}

Departamento de Física Teórica

Facultad de Ciencias Físicas

Universidad Complutense, Madrid, España

\section{Z. Naturforsch. 42 a, 905-906 (1987); received April 25, 1987}

We propose an explicit finite difference scheme to solve operator equations of motion in quantum mechanics and in a quantum scalar field theory.

PACS numbers: 05.50. + q, 02.60. + y, 03.70. + k

Bender and Sharp [1, 2] have proposed a differencing scheme to solve the operator equations in quantum mechanics and in quantum field theory. The main property of this method is that the canonical equal-time commutation relations (ETCR's) are preserved. On the other hand Moncrief [3] pointed out the computational difficulties of the Bender-Sharp differencing scheme due to its implicit character. To avoid these problems, Moncrief has proposed an explicit scheme which also preserves the ETCR's. Later Vázquez [4] suggested two new explicit finite difference schemes, which are related to the one of Moncrief.

The purpose of this paper is to propose an explicit scheme, which is accurate to order $\tau^{4}, \tau$ being the fixed time increment. This scheme represents an improvement of the above explicit schemes because they are accurate only to order $\tau^{2}$.

Let us consider a one-dimensional quantum system

$$
H=p^{2} / 2+V(q) .
$$

The Heisenberg equations of motion are

$$
\mathrm{d} q(t) / \mathrm{d} t=p(t), \quad \mathrm{d} p(t) / \mathrm{d} t=f(q(t))
$$

which also can be written

$$
\mathrm{d}^{2} q(t) / \mathrm{d} t^{2}=f(q(t)) .
$$

$f(q)=-\mathrm{d} V / \mathrm{d} q$, and the operators $p(t)$ and $q(t)$ must satisfy the commutation relation

$$
[q(t), p(t)]=i .
$$

To solve this problem in the interval $[0, T]$, we divide it into $N$ intervals of length $\tau$ and define $q_{n}$ and $p_{n}$ as the operators $q$ and $p$ at time $t=n \tau$.

Reprint requests to Luis Vázquez, Departmento de Física Teórica, Facultad de Ciencias Físicas, Universidad Complutense, 28040 Madrid/España.
The explicit scheme we propose is the following:

$$
\begin{aligned}
& \left(q_{n+1}-q_{n}\right) / \tau=p_{n}, \\
& \frac{-p_{n+1}+15 p_{n}-15 p_{n-1}+}{12 \tau} p_{n-2} \\
& \quad=f\left(\frac{14 q_{n}-q_{n+1}-q_{n-1}}{12}\right),
\end{aligned}
$$

and our problem is to iterate $(5)-(6)$ with the initial condition

$$
\left\{q_{0}, p_{0}\right\} \text { such that }\left[q_{0}, p_{0}\right]=i \text {. }
$$

Also we may reduce the Eqs. $(5)-(6)$ to one equation which only contains the operators $q_{n}$ :

$$
\begin{aligned}
\frac{-q_{n+2}+16 q_{n+1}-30 q_{n}+}{12 \tau^{2}} & =f\left(\frac{14 q_{n-1}-q_{n-2}-q_{n-1}}{12}\right) .
\end{aligned}
$$

This equation is a discrete analogue of (3) and must be iterated with the initial condition

$$
\left\{q_{0}, q_{1}\right\} \text { such that }\left[q_{0}, q_{1}\right]=i \tau,
$$

where the commutation relation is obtained from (5), with $n=0$, and (7).

Let us see the features of the scheme.

1) Accuracy: From calculus [5] we can see that the left side of (8) is a discretization of the second derivative with accuracy of order $\tau^{4}$. Thus the present scheme is more accurate than the explicit ones of [3] and [4].

2) The ETCR's: Let us define, at each step $n \tau$, the average position and momentum operators

$$
\begin{aligned}
& \tilde{q}^{n}=\frac{1}{12}\left(14 q^{n}-q^{n+1}-q^{n-1}\right), \\
& \tilde{p}^{n}=\frac{1}{12}\left(14 p^{n}-p^{n+1}-p^{n-1}\right),
\end{aligned}
$$

then from (5) - (6) and (8) we get

$$
\begin{aligned}
& \frac{\tilde{q}_{n+1}-\tilde{q}_{n}}{\tau}=\tilde{p}_{n}, \\
& \frac{\tilde{p}_{n+1}-\tilde{p}_{n}}{\tau}=f\left(\tilde{q}_{n+1}\right), \\
& \frac{\tilde{q}_{n+1}-2 \tilde{q}_{n}+\tilde{q}_{n-1}}{\tau^{2}}=f\left(\tilde{q}_{n}\right) .
\end{aligned}
$$

If the initial condition is $\left\{\tilde{q}_{0}, \tilde{p}_{0}\right\}$ such that $\left[\tilde{q}_{0}, \tilde{p}_{0}\right]=i$ it is easy to check, in (11) and (12), that

$$
\left[\tilde{q}_{n}, \tilde{p}_{n}\right]=i .
$$

In the same way, when the initial condition is expressed only in terms of the operators $\tilde{q}_{n}$ :

$$
\left\{\tilde{q}_{-1}, \tilde{q}_{0}\right\} \text { such that }\left[\tilde{q}_{-1}, \tilde{q}_{0}\right]=i \tau,
$$

0932-0784 / 87 / 0800-0905 \$01.30/0. - Please order a reprint rather than making your own copy. 
we can see from (13) that

$$
\left[\tilde{q}_{n}, \tilde{q}_{n+1}\right]=i \tau \text {. }
$$

Thus in our scheme the ETCR's are preserved, at each step, by the averaged operators $\tilde{q}_{n}$ and $\tilde{p}_{n}$.

3) Our scheme is not selfstarting, since to iniciate the iteration we need to know either the operators $\left\{q_{0}, p_{0}, p_{-1}, p_{-2}\right\}$ or $\left\{q_{1}, q_{0}, q_{-1}, q_{-2}\right\}$ if the computations are made using $(5)-(6)$ or (8), respectively. However, the data operators are either $\left\{q_{0}, p_{0}\right\}$ or $\left\{q_{0}, q_{1}\right\}$. Let us analyze this problem in terms only of the operators $q_{n}$. In a similar way it can be made for the operators $q_{n}$ and $p_{n}$. We made the simple linear interpolation

$$
\begin{aligned}
& q_{-1}=q_{0}-\left(q_{1}-q_{0}\right)=2 q_{0}-q_{1}, \\
& q_{-2}=q_{0}-2\left(q_{1}-q_{0}\right)=3 q_{0}-2 q_{1} .
\end{aligned}
$$

Thus the following commutation relations are satisfied:

$$
\left[q_{-2}, q_{-1}\right]=i \tau, \quad\left[q_{-1}, q_{0}\right]=i \tau, \quad\left[\tilde{q}_{-1}, \tilde{q}_{0}\right]=i \tau .
$$

then from (19)-(20) we get the equations

$$
\begin{aligned}
& \frac{\tilde{\Phi}_{j}^{n+1}-\tilde{\Phi}_{j}^{n}}{\tau}=\tilde{\Pi}_{j}^{n}, \\
& \frac{\tilde{\Pi}_{j}^{n+1}-\tilde{\Pi}_{j}^{n}}{\tau}=\frac{\tilde{\Phi}_{j+1}^{n+1}-2 \tilde{\Phi}_{j}^{n+1}+\tilde{\Phi}_{j-1}^{n+1}}{h^{2}}+f\left(\tilde{\Phi}_{j}^{n+1}\right)
\end{aligned}
$$

which preserve the ETCR's for the average operators:

$\left[\tilde{\Phi}_{j}^{n}, \tilde{\Phi}_{k}^{n}\right]=0, \quad\left[\tilde{\Pi}_{j}^{n}, \tilde{\Pi}_{k}^{n}\right]=0, \quad\left[\tilde{\Phi}_{j}^{n}, \tilde{\Pi}_{k}^{n}\right]=\frac{i}{h} \delta_{j k}$.

And also we have the discreteness effect

$$
\left[\tilde{\Phi}_{j}^{n}, \tilde{\Phi}_{k}^{n+1}\right]=i \frac{\tau}{h} \delta_{j k} .
$$

As before, we can reduce (19) - (20) to one equation which only contains the operators $\Phi_{j}^{n}$ :

$$
\begin{aligned}
\frac{-\Phi_{j}^{n+2}+16 \Phi_{j}^{n+1}-30 \Phi_{j}^{n}+16 \Phi_{j}^{n-1}-\Phi_{j}^{n-2}}{\tau^{2}} & =f\left(\frac{14 \Phi_{j}^{n}-\Phi_{j}^{n+1}-\Phi_{j}^{n-1}}{12}\right) \\
+ & \frac{14\left(\Phi_{j+1}^{n}-2 \Phi_{j}^{n}+\Phi_{j-1}^{n}\right)-\left(\Phi_{j+1}^{n+1}-2 \Phi_{j}^{n+1}+\Phi_{j-1}^{n+1}\right)-\left(\Phi_{j+1}^{n-1}-2 \Phi_{j}^{n-1}+\Phi_{j-1}^{n-1}\right)}{h^{2}}
\end{aligned}
$$

That allows us to iterate (8).

Now we apply our scheme to a nonlinear quantum scalar field theory in two-dimensional Minkowski space:

$$
\Phi_{t}=\Pi, \quad \Pi_{t}-\Phi_{x x}-f(\Phi)=0 .
$$

We discretize (18) by using a mesh of sizes $\Delta t=\tau$ and $\Delta x=h$ as follows:

$$
\begin{aligned}
& \frac{\Phi_{j}^{n+1}-\Phi_{j}^{n}}{\tau}=\Pi_{j}^{n}, \\
& \frac{-\Pi_{j}^{n+1}+15 \Pi_{j}^{n}-15 \Pi_{j}^{n-1}+\Pi_{j}^{n-2}}{12 \tau} \\
& +\frac{14\left(\Phi_{j+1}^{n}-2 \Phi_{j}^{n}+\Phi_{j-1}^{n}\right)-\left(\Phi_{j+1}^{n+1}\right.}{\Phi_{j}^{n} \text { and } \Pi_{j}^{n} \text { are the field operators }} \\
& \tau, x=j h) \text { let us define in each point of the latti } \\
& \tilde{\Phi}_{j}^{n}=\frac{1}{12}\left(14 \Phi_{j}^{n}-\Phi_{j}^{n+1}-\Phi_{j}^{n-1}\right), \\
& \Pi_{j}^{n}=\frac{1}{12}\left(14 \Pi_{j}^{n}-\Pi_{j}^{n+1}-\Pi_{j}^{n-1}\right) ;
\end{aligned}
$$$$
\frac{-\Pi_{j}^{n+1}+15 \Pi_{j}^{n}-15 \Pi_{j}^{n-1}+\Pi_{j}^{n-2}}{12 \tau}=f\left(\frac{14 \Phi_{j}^{n}-\Phi_{j}^{n+1}-\Phi_{j}^{n-1}}{12}\right)
$$$$
+\frac{14\left(\Phi_{j+1}^{n}-2 \Phi_{j}^{n}+\Phi_{j-1}^{n}\right)-\left(\Phi_{j+1}^{n+1}-2 \Phi_{j}^{n+1}+\Phi_{j-1}^{n+1}\right)-\left(\Phi_{j+1}^{n-1}-2 \Phi_{j}^{n-1}+\Phi_{j-1}^{n-1}\right)}{12 h^{2}}
$$

where $\Phi_{j}^{n}$ and $\Pi_{j}^{n}$ are the field operators at the point $(t=n \tau, x=j h)$.

Now let us define in each point of the lattice the average operators: which shows that the accuracy of the scheme is of order $\tau^{4}$.

Finally, the problem of starting the iteration can be solved in the same way as before.

\section{Acknowledgements}

I thank the Comisión Asesora de Investigación Científica y Técnica for financial support (Grant $N^{\circ} 2140 / 83$ ).
[1] C. M. Bender and D. H. Sharp, Phys. Rev. Lett. 50, 1535 (1983).

[2] C. M. Bender, K. A. Milton and D. H. Sharp, Phys. Rev. Lett. 51, 1815 (1983).

[3] V. Moncrief, Phys. Rev. D 28, 2485 (1983).

[4] L. Vázquez, Z. Naturforsch. 41 a, 788 (1986).

[5] J. P. Nougier, Méthodes de Calcul Numérique, Masson, Paris 1983. Chapter 3. 